Original Research Paper

\title{
Large Scale Synthesis of ZnO Nanostructures of Different Morphologies through Solvent-free Mechanochemical Synthesis and their Application in Photocatalytic Dye Degradation
}

\author{
${ }^{1}$ Raúl Sánchez Zeferino, ${ }^{2}$ Jesús Alberto Ramos Ramón, \\ ${ }^{2}$ Ma. Eunice de Anda Reyes, ${ }^{2}$ Rutilo Silva González and ${ }^{2}$ Umapada Pal \\ ${ }^{1}$ Departamento de Física, Universidad de Sonora, Apdo. Postal 1626, Col. Centro, Hermosillo 83000, Sonora, Mexico \\ ${ }^{2}$ Instituto de Física, Benemérita Universidad Autónoma de Puebla, Apdo. Postal J-48, Puebla, Pue. 72570, Mexico
}

\section{Article history}

Received: 28-10-2015

Revised: 08-12-2015

Accepted: 13-01-2016

Corresponding Author:

Umapada $\mathrm{Pal}$

Instituto de Física, Benemérita

Universidad Autónoma de Puebla,

Apdo. Postal J-48, Puebla, Pue.

72570, Mexico

Email: upal@ifuap.buap.mx

\begin{abstract}
Well crystalline, near-stoichiometric zinc oxide nanostructures of different morphologies are fabricated in large scale, utilizing a simple, cost effective mechanochemical synthesis in absence of solvent. Effects of ionic and nonionic surfactants along with the concentration of hydrolyzing agent on the evolution of nanostructure morphology have been studied. It has been observed that while a cationic surfactant such as Cetyltrimethylammonium Bromide (CTAB) favors the nanostructures to growth along their polar c-axis, a nonionic surfactant such as Polyvinylpyrrolidone (PVP) reduces their preferred c-axis growth. Increase of hydrolyzing agent in the reaction mixture enhances the growth rate of the nanostructures. The nanostructures have been tested for photodegradation of anionic dye molecules such as Methylene Blue (MB). All the nanostructures manifest high photocatalytic performance. Apart from morphology, the specific surface area, crystal plane orientation and the concentration of basic sites at surface are seen to contribute significantly to the photocatalytic performance of the zinc oxide nanostructures.
\end{abstract}

Keywords: ZnO, Nanostructures, Mechanochemical Synthesis, Photocatalysis, Dye Degradation

\section{Introduction}

Recent thrust of metal oxide nanostructure research is driven by their application potentials in diverse fields such as gas sensing (Hsu et al., 2008; Kumar et al., 2015; Wang et al., 2006; 2012; Xu et al., 2000), optoelectronic device fabrication (Hsu et al., 2008; Olson et al., 2007; Valerini et al., 2008; Zimmler et al., 2008), catalysis (Matsamura and Ishibe, 2009; Lorenz et al., 2013; Spencer 1999) photocatalysis (Jia et al., 2015; Morales Flores et al., 2014; Ruiz Peralta et al., 2012; Yang et al., 2015) and dye adsorption (Chen et al., 2015; Khosla et al., 2015; Norman, 1970). Due to high thermal and chemical stability and reasonable biocompatibility, the application of metal oxide nanostructures extended further to biology and medicine (Hahn et al., 2012; Hilger and Kaiser, 2012; Solanki et al., 2011). While for each of these applications metal oxide nanostructures of specific nature and characteristics such as size, composition, nature of dopant and morphology are frequently preferred, apart from some basic characteristics such as band gap energy, excitonic energy, photoresponse and specific surface area, the role of other characteristics has not been associated to their performances with certainty. To elucidate the facts, we can consider a specific material, such as zinc oxide, which is a metal oxide semiconductor of high band gap energy (3.37 eV at room temperature) and high excitonic energy $(60 \mathrm{meV})$. In nanostructure forms, it is very much suitable for fabricating optoelectronic and piezoelectric devices (Jalali et al., 2013; Solanki et al., 2011; Wang and Song, 2006), photocatalytic degradation of organic materials (Morales Flores et al., 2014; Ruiz Peralta et al., 2012) and catalytic supports 
(Lorenz et al., 2013; Matsamura and Ishibe, 2009; Spencer, 1999). ZnO nanostructures of different morphologies and sizes have been fabricated using a vast number of chemical and physical methods and the roles of their morphology, defect structure and surface area have been studied extensively, especially in the context of later applications (Jalali et al., 2013; Pan et al., 2014; Ruiz Peralta et al., 2012; Wang and Song, 2006). While through physical evaporation process, $1 \mathrm{D} \mathrm{ZnO}$ nanostructures such as nanorods, nanowires and nanobelts have been fabricated (An et al., 2013; He et al., 2006; Wang et al., 2005; 2004a; 2004b), through chemical processes such as sol-gel (Tak and Yong, 2005), hydrothermal and other solution based synthesis (Chen et al., 2000; Ni et al., 2005; Shao et al., 2008; Xie et al., 2011), ZnO nanostructures of a large variety of morphologies have been fabricated successfully. Utilizing amine-assisted nonhydrolyzing synthesis method, Zhang et al. (2007) could fabricate $\mathrm{ZnO}$ nanostructures of different morphologies using zinc acetate precursor. On the other hand, utilizing the simple and cheap mechanochemical technique, $\mathrm{ZnO}$ nanostructures of different morphologies (Anand et al., 2014; Tsuzuki and McCormick, 2001; 2004; Shen et al., 2003) could be synthesized with good control over their shape and size. In fact, a vast number of reports have been published on the morphology control of $\mathrm{ZnO}$ nanostructures through hard and soft chemical routs and their photocatalytic performances for organic dye degradation under UV illumination. While controlled fabrication of $\mathrm{ZnO}$ nanostructures of different morphologies and specific surface areas through cheap and soft chemical routs remains a challenge, understanding the roles of morphology, surface area, defect content and surface acid/base sites on their catalytic and photocatalytic performance is the other task, essential for fabricating suitable $\mathrm{ZnO}$ nanostructures for efficient environmental applications.

In the present article, we demonstrate the fabrication of $\mathrm{ZnO}$ nanostructures of different morphologies in large scale through a solvent-free soft mechanochemical process, utilizing ionic and non-ionic surfactants (structure driving agents) and $\mathrm{NaOH}$ as precipitating/hydrolyzing agent. Effects of morphology, surface area and surface acid/base sites on their photocatalytic dye degradation performance have been studied applying Methylene Blue (MB) as a test dye. It has been demonstrated that the surfactant and concentration of hydrolyzing agent both play important roles for defining the final morphology of $\mathrm{ZnO}$ nanostructures. The roles of surfactant and hydrolyzing agent on the evolution of morphology and photocatalytic performance of mechanochemically grown $\mathrm{ZnO}$ nanostructures have been discussed.

\section{Experimental}

\section{Reagents and Solvents}

Analytical grade zinc acetate dihydrate $\left[\mathrm{Zn}\left(\mathrm{CH}_{3} \mathrm{COO}\right)_{2} \cdot 2 \mathrm{H}_{2} \mathrm{O}\right]$, sodium hydroxide $(\mathrm{NaOH})$, Cetyltrimethylammonium Bromide (CTAB) and polyvinylpyrrolidone (PVP, MW 40,000) were purchased from Sigma-Aldrich, Mexico and used as received without further purification.

\section{Preparation of $\mathrm{ZnO}$ Nanostructures using CTAB and PVP}

$\mathrm{ZnO}$ nanostructures were synthetized through mechanochemical process following the procedure reported by Anand et al. (2014) with some modifications. Briefly, Zinc Acetate dihydrate (ZnAc) and CTAB or PVP in 2:1 molar ratio were mixed in a ceramic mortar for about $30 \mathrm{~min}$. After that, a certain amount $\mathrm{NaOH}$ was added to the above mixture and manually grinded for another $30 \mathrm{~min}$ at room temperature. The molar ratio of $\mathrm{ZnAc}$ and $\mathrm{NaOH}$ in the mixture was maintained either 1:3 or 1:9. The obtained white pastes were dispersed in water and washed with ethanol and water several times by centrifugation $(8000$ $\mathrm{rpm}, 10 \mathrm{~min}$ ). Obtained samples were dried at room temperature and annealed thereafter at $350^{\circ} \mathrm{C}$ for $2 \mathrm{~h}$ in air. Utilizing $40 \mathrm{mmol}$ of zinc acetate precursor, about 3.0-3.2 g of $\mathrm{ZnO}$ nanostructures were obtained for each of the samples. The synthesis conditions used for fabricating different $\mathrm{ZnO}$ nanostructures and their nomenclatures are given in Table 1.

\begin{tabular}{|c|c|c|c|c|c|c|c|}
\hline Sample name & $\begin{array}{l}\mathrm{ZnAc} / \mathrm{NaOH} \\
\text { (molar ratio) }\end{array}$ & $\begin{array}{l}\text { Surfactant } \\
\text { used }\end{array}$ & Morphology & $\begin{array}{l}\text { EDS estimated } \\
\mathrm{Zn:O} \text { (at. ratio) }\end{array}$ & $\begin{array}{l}\text { BET surface } \\
\text { area }\left(\mathrm{m}^{2} / \mathrm{g}\right)\end{array}$ & $\begin{array}{l}\text { BET Av. pore } \\
\text { diameter (nm) }\end{array}$ & $\begin{array}{l}\text { XRD estimated Av. } \\
\text { crystallite size (nm) }\end{array}$ \\
\hline $\mathrm{ZnO}-1$ & $1: 3$ & - & $\begin{array}{l}\text { elongated } \\
\text { particles }\end{array}$ & 1.05 & 16.35 & 63.1 & 32.7 \\
\hline $\mathrm{ZnO}-2$ & $1: 3$ & $\begin{array}{l}\text { CTAB } \\
(5.4 \mathrm{mmol})\end{array}$ & nanorods & 1.03 & 8.72 & 30.1 & 38.4 \\
\hline $\mathrm{ZnO}-3$ & $1: 9$ & $\begin{array}{l}\text { CTAB } \\
(5.4 \mathrm{mmol})\end{array}$ & $\begin{array}{l}\text { Flower consisting } \\
\text { nanorods (echinoids) }\end{array}$ & 1.02 & 8.35 & 46.8 & 35.2 \\
\hline $\mathrm{ZnO}-4$ & $1: 3$ & $\begin{array}{l}\text { PVP } \\
(0.05 \mathrm{mmol})\end{array}$ & spherical particles & 1.04 & 12.36 & 80.5 & 36.2 \\
\hline $\mathrm{ZnO}-5$ & $1: 9$ & $\begin{array}{l}\text { PVP } \\
(0.05 \mathrm{mmol})\end{array}$ & $\begin{array}{l}\text { flowers } \\
\text { consisting petals }\end{array}$ & 1.03 & 6.90 & 40.4 & 33.8 \\
\hline
\end{tabular}


The samples were prepared at Zinc Acetate (ZnAc) and $\mathrm{NaOH}$ molar ratios of 1:3 and 1:9 for a fixed molar content of surfactants CTAB and PVP to study the effects of surfactant and $\mathrm{NaOH}$ concentration on the morphology and photocatalytic behaviors of the fabricated nanostructures. As the growth rate of $\mathrm{ZnO}$ nanostructures and their morphology are known to be driven by the surface energy of their crystal facets or planes, which generally get modified by surfactants apart from the $\mathrm{pH}$ of the reaction (Xie et al., 2009; Jang et al., 2009), we used CTAB and PVP as ionic (cationic) and non-ionic surfactants, respectively, to observe the effect of surfactant nature on morphology evolution of $\mathrm{ZnO}$ nanostructures.

\section{Characterization of the $\mathrm{ZnO}$ Nanostructures}

The crystallinity and structural phase of the nanostructures were analyzed through powder X-Ray Diffraction (XRD, Bruker D8 Discover) and micro Raman spectroscopy (Horiba Yovin Lab RAM HR). While the XRD patterns of the samples were recorded in $10-70^{\circ}$ range utilizing $\mathrm{CuK} \alpha(\lambda=1.5406 \AA)$ radiation in $0.02^{\circ} 2 \theta$ steps, their room temperature Raman spectra were recorded using the $633.8 \mathrm{~nm}$ line $(10 \mathrm{~mW}, 1.0 \mathrm{~mm}$ spot size) of a He-Ne laser as excitation source and a thermoelectrically cooled charge-coupled device as detector. For morphology and elemental analysis, a JEOL JSM-7800F Field-Emission Scanning Electron Microscope (FESEM) with Oxford X-Max analytical accessory was used. A JEOL JEM 2100F field emission transmission electron microscope (FE-TEM) operating at $200 \mathrm{kV}$ was utilized to study the crystal structure and growth induced defects in the nanostructures. To estimate the specific surface area and average pore size in the nanostructures, their adsorption-desorption isotherms at $77 \mathrm{~K}$ were recorded in a Belsorp-Mini II (BEL Japan, Inc.) analyzer. The samples were degassed at $250^{\circ} \mathrm{C}$ for $5 \mathrm{~h}$ under vacuum prior to their analysis. The specific surface area and average pore diameter in the samples were estimated utilizing Brunauer-EmmettTeller (BET) procedure (Brunauer et al., 1938).

\section{Photocatalytic Evaluation of the $\mathrm{ZnO}$ Nanostructures}

The photocatalytic responses of the nanostructures were recorded in a Shimadzu UV-3101 PC UV-Vis-NIR double-beam spectrophotometer. The tests were performed in aqueous solution using methylene blue (MB, $\mathrm{C}_{16} \mathrm{H}_{18} \mathrm{ClN}_{3} \mathrm{~S} \cdot 3 \mathrm{H}_{2} \mathrm{O}$, Sigma-Aldrich) as a test contaminant. A cylindrical jacketed glass mini-reactor of $500 \mathrm{~mL}$ capacity with $50 \mathrm{~mm}$ internal diameter and about $120 \mathrm{~mm}$ of height was utilized to perform batch reactions. Room temperature $\mathrm{MB}$ photodegradation rate of the catalysts was monitored by dispersing $20 \mathrm{mg}$ of powder $\mathrm{ZnO}$ sample in $40 \mathrm{~mL}$ of $\mathrm{MB}$ solution (10 ppm).
The catalyst-containing reaction mixture under magnetic agitation was illuminated by a $10 \mathrm{~W}$ ultraviolet LED lamp (model JX-10UV9X1B365) of $365 \mathrm{~nm}$ emission. The lamp was placed at the top of the reactor, about 13 $\mathrm{cm}$ above the test solution surface. The temperature of the reaction mixture was maintained fixed at $25^{\circ} \mathrm{C}$ by circulating cold water through the jacket of the reactor. Oxygen was bubbled through the reaction solution under UV illumination.

The MB concentration in the reaction mixture was monitored by a computer-controlled spectrophotometer at regular intervals, withdrawing each time about $3.5 \mathrm{~mL}$ of the solution (aliquot) from the reactor. To separate the dispersed catalyst from the aliquot, a reusable syringe filter holders (z268410) with nitrocellulose membrane filter of $0.22 \mathrm{~mm}$ pore size was utilized. From the absorbance spectra of $\mathrm{MB}$ recorded at different time intervals, the concentrations of $\mathrm{MB}$ in the reaction solution were estimated using a pre-calibrated concentration curve. The MB concentration in the reaction mixture was determined from the intensity of the most prominent absorption band of MB around $664.5 \mathrm{~nm}$.

\section{Results and Discussion}

Figure 1 presents the X-ray diffraction patterns of the samples prepared at different $\mathrm{ZnAc:NaOH}$ ratios in absence and presence of surfactants (Table 1). As can be seen, all the samples revealed well resolved, high intensity diffraction peaks correspond to crystalline $\mathrm{ZnO}$ in hexagonal wurtzite phase (JCPDS card no. 79-0207). The relative intensity of the main diffraction peaks like (100), (002) and (101) varied a little from sample to sample. No significant shift in the position of the diffraction peaks was observed for the samples prepared at different precursor- $\mathrm{NaOH}$ molar ratios or for different surfactants. Utilizing the most intense (101) diffraction peak, average grain size $(\mathrm{P})$ in the $\mathrm{ZnO}$ nanostructures was estimated using the Debye Scherrer equation (Cullity and Stock, 1956):

$$
P=\frac{K \lambda}{\beta \cos \theta}
$$

where, $K$ is the shape factor considered to be $0.9, \lambda$ is the $\mathrm{X}$-ray excitation wavelength $(\lambda=1.5406 \AA), \beta$ is the FWHM of the (101) peak and $\theta$ is the Bragg angle. Estimated average grain size values for the $\mathrm{ZnO}$ nanostructures are presented in Table 1. In general, the presence of surfactant (of either nature) enhances the average grain size of the nanostructures. However, apparently the nature of surfactant does not affect their crystallite size. A decrease of average grain size for the nanostructures prepared with higher $\mathrm{NaOH}$ content (higher $\mathrm{pH}$ of the reaction) is probably due to higher growth rate of the nanostructures at higher reaction $\mathrm{pH}$. 


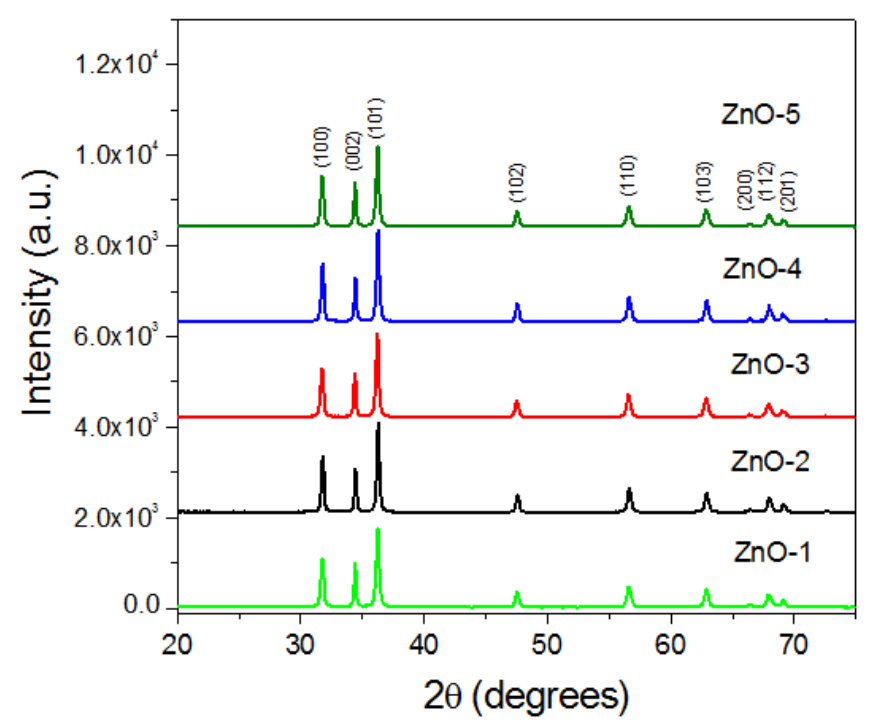

Fig. 1. XRD patterns of the mechanochemically fabricated $\mathrm{ZnO}$ nanostructures utilizing CTAB or PVP as surfactant

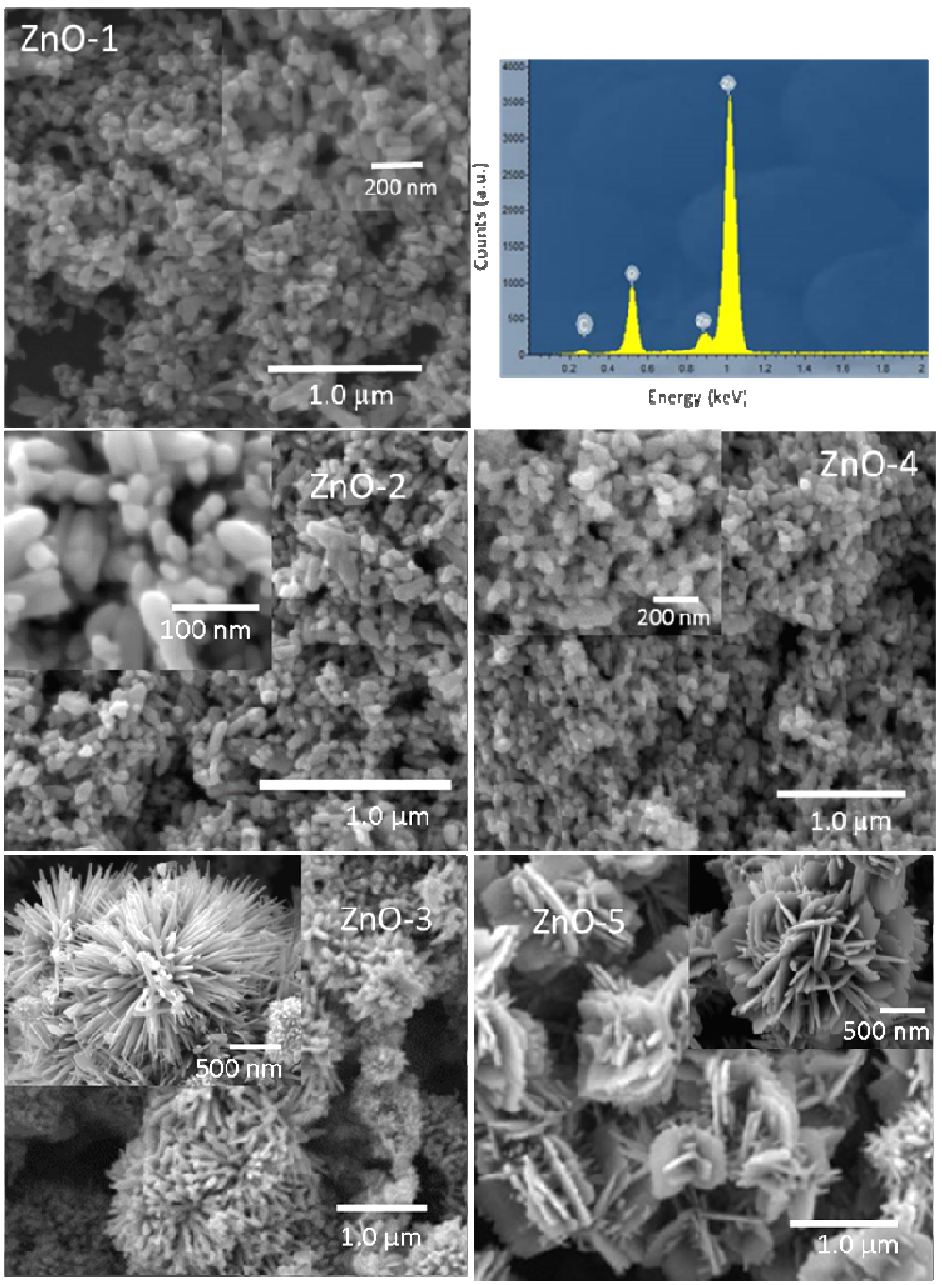

Fig. 2. Typical SEM images of the $\mathrm{ZnO}$ nanostructures fabricated by mechanochemical synthesis, along with their typical EDS spectrum 
As can be seen from Fig. 2, both the nature of surfactant and hydrolyzer concentration have strong effect on the final morphology of $\mathrm{ZnO}$ nanostructures. While in absence of any surfactant, a molar ratio 1:3 of $\mathrm{ZnAc}$ and $\mathrm{NaOH}$ produces elongated nanoparticles (Fig. 2, sample $\mathrm{ZnO}-1$ ) of about $77 \mathrm{~nm}$ average (c.a.) size, presence of CTAB (cationic surfactant) enhances the growth of $\mathrm{ZnO}$ along polar c-axis, resulting rod-like 1D nanostructures of about $300 \mathrm{~nm}$ average (c.a.) length and $50 \mathrm{~nm}$ average diameter (c.a.). On the other hand, the effect of reaction $\mathrm{pH}$ on morphology evolution of $\mathrm{ZnO}$ nanostructures is very prominent for both the surfactants. As can be seen from the SEM images of the samples $\mathrm{ZnO}-3$ and $\mathrm{ZnO}-5$ prepared at 1:9 precursorhydrolyzer molar ratio using CTAB and PVP as surfactant, respectively, the $\mathrm{pH}$ of the reaction affects the morphology of $\mathrm{ZnO}$ nanostructures severely. While the former sample revealed echinoid like (flower consisting nanorods emanating from a common point) morphology, the later sample revealed rose-like (flower consisting petals) morphology.

The evolution of very distinct morphologies in these two samples clearly demonstrates that both the $\mathrm{pH}$ of the reaction and the nature of surfactant define the final morphology of $\mathrm{ZnO}$ nanostructures in mechanochemical synthesis.

The observed morphologies for the samples prepared at different reaction conditions can be understood considering the polar nature of $\mathrm{ZnO}$, roles of hydrolyzing agent and surfactant on the modification of surface energy of its different facets or crystal planes.

The commonly accepted growth process of $\mathrm{ZnO}$ crystallites in basic aqueous medium can be described through the reactions (Huang et al., 2010; Morales Flores et al., 2014):

$$
\begin{aligned}
& \mathrm{Zn}^{2+}+2 \mathrm{OH}^{-} \rightarrow \mathrm{Zn}(\mathrm{OH})_{2} \downarrow \\
& \mathrm{Zn}(\mathrm{OH})_{2}+2 \mathrm{OH}^{-} \rightarrow \mathrm{Zn}(\mathrm{OH})_{4}{ }^{2-} \\
& \mathrm{Zn}(\mathrm{OH})_{4}{ }^{2-} \rightarrow \mathrm{ZnO}+\mathrm{H}_{2} \mathrm{O}+2 \mathrm{OH}^{-}
\end{aligned}
$$

In basic solution, where $\mathrm{OH}$ ions are abundant, first the $\mathrm{Zn}^{2+}$ ions form $\mathrm{Zn}(\mathrm{OH})_{2}$ precipitate following the reaction (2). For highly basic solution, the formed $\mathrm{Zn}(\mathrm{OH})_{2}$ precipitate dissolves almost immediately by reacting with excess $\mathrm{OH}$ ions, forming $\mathrm{Zn}(\mathrm{OH})_{4}{ }^{2-}$ ions following reaction (3). The $\mathrm{Zn}(\mathrm{OH})_{4}{ }^{2-}$ growth units combine with each other and dehydrate to form $\mathrm{ZnO}$ nuclei simultaneously (Equation 4). Through a selfassembly or oriented attachment of the nuclei, elongated $\mathrm{ZnO}$ nanostructures are formed with preferred $c$-axis orientation. In the case of solvent-free mechanochemical synthesis, absence of a solvent inhibits the formation of metal ions and hence the formation of $\mathrm{Zn}(\mathrm{OH})_{2}$. The reaction in highly basic condition favors a direct formation of $\mathrm{Zn}(\mathrm{OH})_{4}{ }^{2-}$ and its fast dehydration to form $\mathrm{ZnO}$ nuclei. Although the growth habit of $\mathrm{ZnO}$ crystal is mainly driven by its intrinsic structure, external parameters such as the presence of surfactant and $\mathrm{pH}$ of the reaction have significant effects (Bai et al., 2010). Addition of cationic CTAB in the reaction solution not only reduces its surface tension, reducing the energy of formation of $\mathrm{ZnO}$, induces the formation of $\mathrm{CTA}^{+}-\mathrm{Zn}(\mathrm{OH})_{4}{ }^{2-}$ ion-pair, enhancing the transport of $\mathrm{Zn}(\mathrm{OH})_{4}{ }^{2-}$ units (Yan et al., 2001) and hence the growth rate of $\mathrm{ZnO}$ nanostructures. As we see in the Fig. 2 (sample $\mathrm{ZnO}-2$ ), incorporation of $\mathrm{CTAB}$ in the $\mathrm{ZnAc}-\mathrm{NaOH}$ mixture of $1: 3$ molar ratio results $\mathrm{ZnO}$ nanorods of longer dimension and smaller diameters than the elongated nanoparticles formed in its absence (sample $\mathrm{ZnO}-1$ ). Incorporation of additional $\mathrm{NaOH}$ in the reaction mixture results the formation of longer $\mathrm{ZnO}$ nanorods emanating from a common point of nucleation as seen for the sample $\mathrm{ZnO}-3$. In fact, addition of excess $\mathrm{NaOH}$ in the reaction mixture enhances the hydrolysis and condensation rates in presence of water molecules (whatever adsorbed by $\mathrm{NaOH}$ during mechanochemical processing). Such uncontrolled and unselective hydrolysis/condensation leads to the formation of highly branched or flower-like structures, irrespective of the nature of added surfactant. In the case of PVP, as it is a nonionic surfactant, its addition in reaction mixture reduces surface energy of all the crystal faces of $\mathrm{ZnO}$ nuclei due to nonselective adherence, producing nanostructures of spherical morphology as seen for the sample $\mathrm{ZnO}-4$. Again, the incorporation of additional $\mathrm{NaOH}$ in the reaction mixture causes a reduction of surface tension, enhancing the growth rate of $\mathrm{ZnO}$. However, in contrast to the earlier case, where CTAB was utilized as surfactant, due to nonionic nature of PVP, no ionpair is formed. Instead, the excess $\mathrm{OH}^{-}$ions get attached to the polar surface of positive charge, i.e. the $<001>$ face, probably forming a negatively charged dielectric double-layer, hindering its further growth. As a result, the growth of $\mathrm{ZnO}$ along its $c$ axis reduces and the planer or flatter nanostructures of plate-like or sheet-like morphology evolve. Again, an uncontrolled hydrolysis/condensation at high $\mathrm{NaOH}$ concentration leads to the formation of flower-like aggregates of thin sheets. In fact, both the evolution of rod-like and plate-like $\mathrm{ZnO}$ nanostructures induced by surfactant (Li et al., 2008) and the $\mathrm{pH}$ of reaction mixture (Wahab et al., 2009) have been reported in the literature for hydrothermal and precipitation synthesis, respectively.

To study the crystallinity and lattice defects, high resolution TEM images of the typical rod-shaped ( $\mathrm{ZnO}$ - 
2), spherical ( $\mathrm{ZnO}-4)$ and petal-shaped $(\mathrm{ZnO}-5) \mathrm{ZnO}$ nanostructures have been recorded (Fig. 3). As can be seen from Figure 3, all the nanostructure are well crystalline with occasional presence of lattice distortion and point defects (indicated by red arrows). Fast Fourier Transform (FFT) of selected areas of the HRTEM images of all three sample (insets of Fig. 3) revealed well defined points patterns correspond to their crystalline nature. It is interesting to note that the rod-shaped nanostructures (sample $\mathrm{ZnO}-2$ ) contain higher structural defects than the spherical (sample $\mathrm{ZnO}-4$ ) and petalshaped (sample $\mathrm{ZnO}-5$ ) nanostructures. The higher defect content in the $\mathrm{ZnO}-2$ sample is also revealed in its
FFT pattern where the diffraction spots are elongated due to structural disorder.

The crystallinity and lattice defects of the nanostructures were studied further recording their room temperature Raman spectra presented in Fig. 4. To understand the effect of surfactant and hydrolyzing agent $(\mathrm{NaOH})$ separately, the results are presented in two groups: Samples prepared using CTAB (Fig. 4a) and the samples prepared with PVP (Fig. 4b). Nanostructures of both the groups revealed well resolved Raman bands corresponding to fundamental and multiphonon modes of crystalline $\mathrm{ZnO}$.
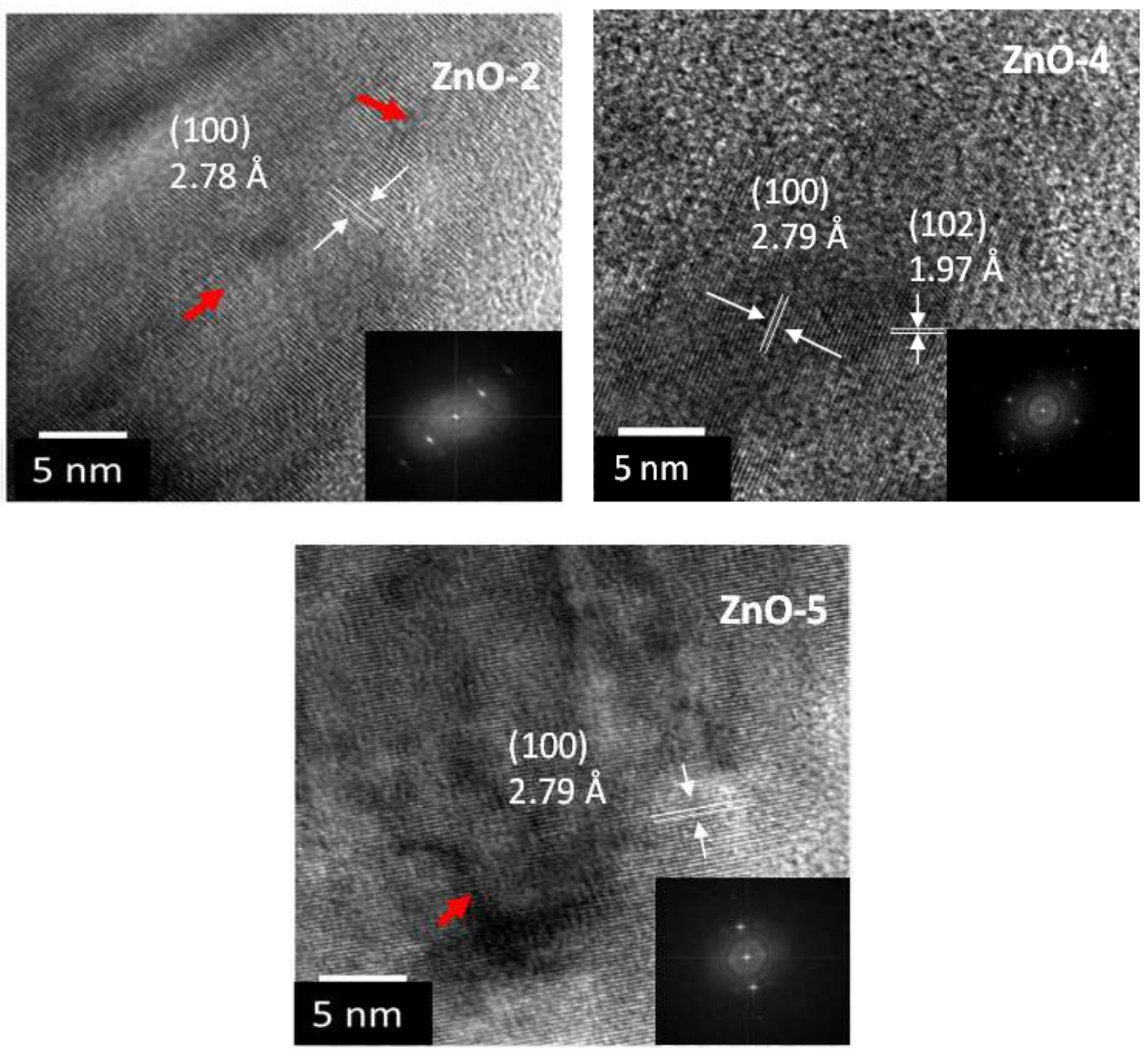

Fig. 3. Typical high resolution TEM micrographs of the rod-shaped ( $\mathrm{ZnO}-2)$, spherical ( $\mathrm{ZnO}-4)$ and petal-shaped (ZnO-5) $\mathrm{ZnO}$ nanostructures and their corresponding FFTs (insets). Defects like vacancies and lattice distortion are shown by arrows in red color 

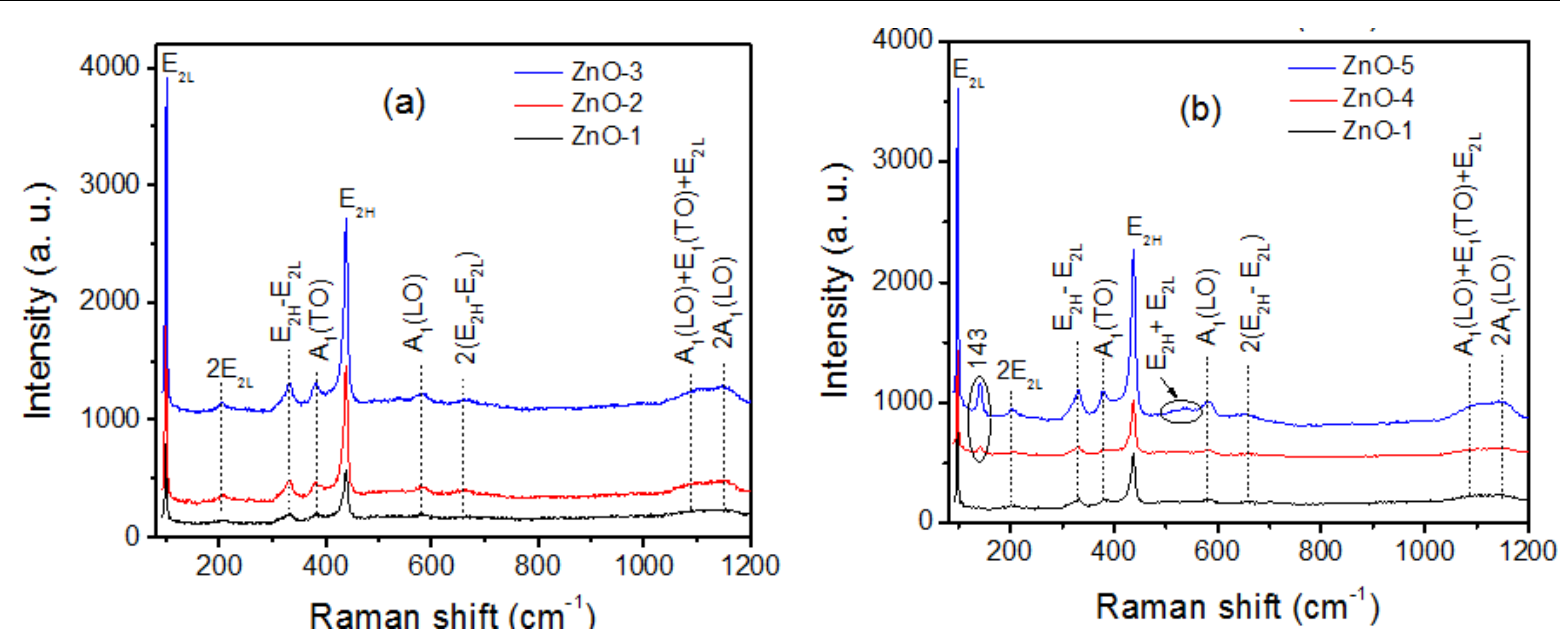

Fig. 4. Room temperature Raman spectra of the $\mathrm{ZnO}$ nanostructures synthetized using (a) CTAB and (b) PVP as surfactant

The intense bands revealed around 100 and $439 \mathrm{~cm}^{-1}$ correspond to the $\mathrm{E}_{2 \mathrm{~L}}$ and $\mathrm{E}_{2 \mathrm{H}}$ modes of nonpolar optical phonons, respectively, characteristic of wurtzite hexagonal ZnO (Morkoç and Özgür, 2008). The Raman bands appeared around 381 and $582 \mathrm{~cm}^{-1}$ are the transverse optical (TO) and longitudinal optical (LO) modes of polar $A_{1}$ mode. Raman spectra of all the samples revealed two weak bands around 204 and 1150 $\mathrm{cm}^{-1}$, which correspond to second-order processes, attributed to $2 \mathrm{E}_{2 \mathrm{~L}}$ and $2 \mathrm{~A}_{1}(\mathrm{LO})$ phonon modes of $\mathrm{ZnO}$, respectively. Moreover, all the Raman bands associated to common multi-phonon processes in $\mathrm{ZnO}$ such as $\mathrm{E}_{2 \mathrm{H}^{-}}$ $\mathrm{E}_{2 \mathrm{~L}}\left(\right.$ at $\left.333 \mathrm{~cm}^{-1}\right), 2\left(\mathrm{E}_{2 \mathrm{H}}-\mathrm{E}_{2 \mathrm{~L}}\right)$ (at $665 \mathrm{~cm}^{-1}$ ) and $\mathrm{A}_{1}(\mathrm{LO})+\mathrm{E}_{1}(\mathrm{TO})+\mathrm{E}_{2 \mathrm{~L}}\left(\right.$ at $1090 \mathrm{~cm}^{-1}$ ) were evolved in the $\mathrm{ZnO}$ nanostructures, demonstrating clearly their high crystalline quality. For the samples synthetized using PVP as surfactant, there appeared two weaker bands around 538 and $143 \mathrm{~cm}^{-1}$, which did not appear for the samples prepared with CTAB. While the earlier band is associated to $\mathrm{E}_{2 \mathrm{H}}+\mathrm{E}_{2 \mathrm{~L}}$ multiphonon in $\mathrm{ZnO}$ lattice, the later band has been found at low temperature microRaman spectra of $\mathrm{ZnO}$ and assigned to intrinsic defects associated with synthesis process (Cuscó et al., 2007).

As one of the aims of this study is to utilize fabricated $\mathrm{ZnO}$ nanostructures as photocatalyst, their surface area and texture have been estimated form nitrogen adsorption-desorption isotherms at $77 \mathrm{~K}$. The adsorption-desorption isotherms of the samples presented in Fig. 5 are typical type III isotherms in Brunauer classification, with no limiting adsorption at high relative pressure. The hysteresis loops can be ascribed to type $\mathrm{H} 3$ of mesoporous material according to the IUPAC classification (Lu et al., 2012). Estimated BET specific surface areas of the nanostructures are presented in Table 2. As we see, the estimated specific surface area of the samples vary in between 6.9 to 16.35 $\mathrm{m}^{2} \mathrm{~g}^{-1}$; highest for the sample $\mathrm{ZnO}-1$ (prepared with no surfactant, $\mathrm{ZnAc:NaOH}=1: 3$ ) and lowest for $\mathrm{ZnO}-5$ (prepared with PVP, $\mathrm{ZnAc:NaOH}=1: 9$ ). On the other hand, the average pore size in the nanostructures estimated using BET analysis varied in between 30.1 (sample $\mathrm{ZnO}$ 2) and $80.5 \mathrm{~nm}$ (sample $\mathrm{ZnO}-4$ ). As it can be noticed, the estimated average pore size of most of the samples is beyond mesoporous limit $(2-50 \mathrm{~nm})$. Such high average pore size values estimated for the nanostructures clearly demonstrate that they correspond to inter-particle spaces, rather than the porosity of individual nanostructure. In fact, the pore size distribution of all the samples through Barrett-Joyner-Halenda (BJH) (Barrett et al., 1951) analysis revealed a prominent peak at around $1.64 \mathrm{~nm}$ pore radius, along with some broader distributions at higher values (insets of Fig. 4).

As it is well known, on illumination with high energy light, the valance band electrons of $\mathrm{ZnO}$ get promoted to its conduction band, creating electron-hole pairs (Equation 5), which diffuse to the surface of the semiconductor:

$Z n O+h v \rightarrow Z n O+h_{v B}^{+}+e_{C B}^{-}$

The photo-generated holes near the nanostructure surface react with surface $\mathrm{OH}^{-}$groups and $\mathrm{H}_{2} \mathrm{O}$ molecules to produce $\mathrm{HO}^{\circ}$ radicals (Equation 6 and 7), which get adsorbed at the catalyst surface. The surface adsorbed $\mathrm{HO}^{\circ}$ radicals are strong oxidizing agents, which react with surface adsorbed organic dye molecules such as MB, mineralizing them partially or completely following the reaction (Equation 8):

$h_{V B}^{+}+H_{2} \mathrm{O} \rightarrow \mathrm{OH}^{-}+H^{+}$

$h_{V B}^{+}+\mathrm{OH}^{-} \rightarrow H O^{\bullet}$

$H O^{\bullet}+M B \rightarrow$ degradation of $M B$ 

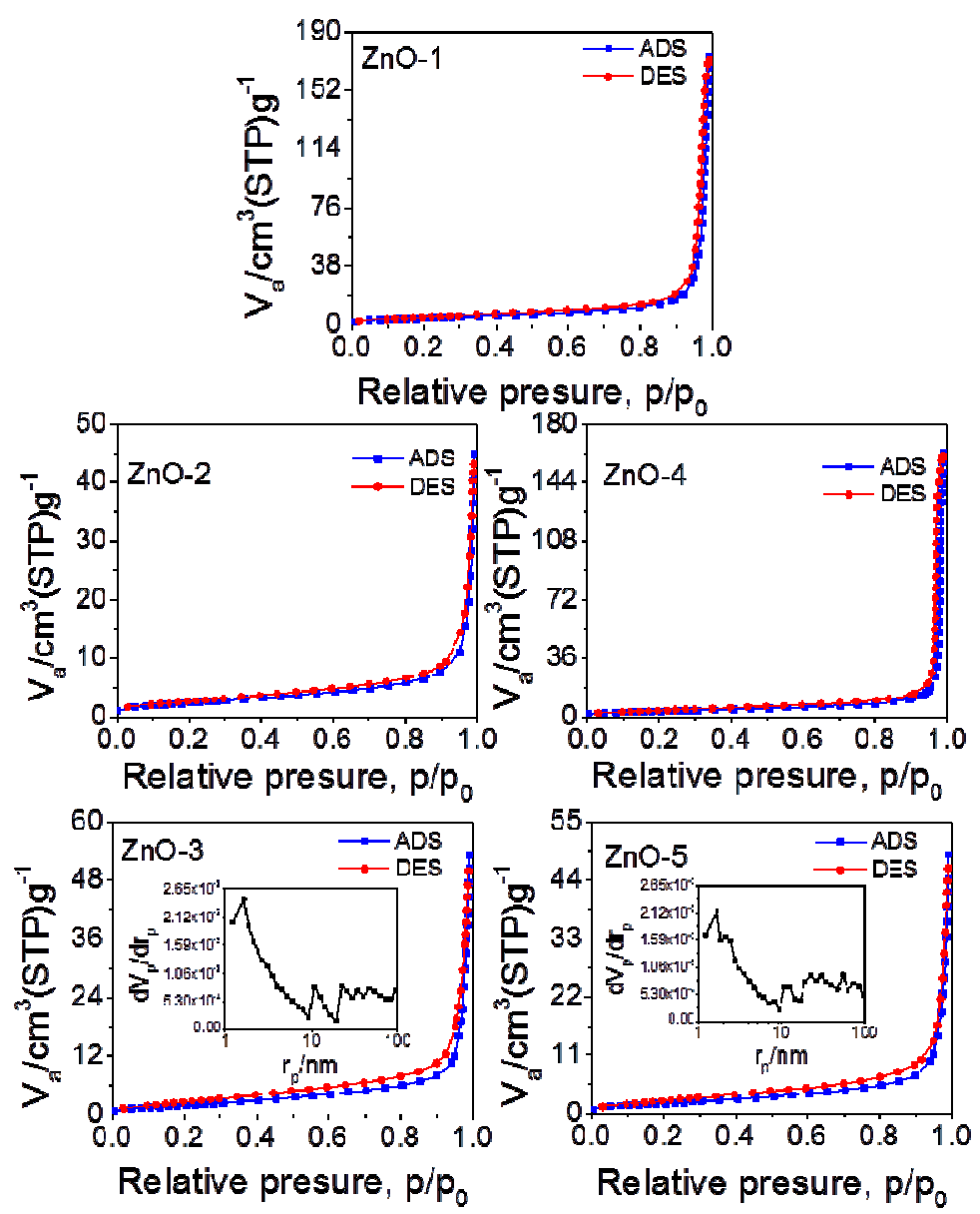

Fig. 5. $\mathrm{N}_{2}$ adsorption-desorption isotherms of the $\mathrm{ZnO}$ nanostructures measured at $77 \mathrm{~K}$. The insets are the pore size distributions (BJH-plots) of corresponding samples

Table 2. Pseudo-first-order rate constant $(k)$, the half-life $\left(t_{1 / 2}\right)$ and $R^{2}$ of MB degradation by the $\mathrm{ZnO}$ nanostructures fabricated by mechanochemical synthesis with comparison with their specific surface area and surface basics sites

\begin{tabular}{llllll}
\hline Sample & $\begin{array}{l}\text { BET surface } \\
\text { area }\left(\mathrm{m}^{2} / \mathrm{g}\right)\end{array}$ & $\begin{array}{l}\text { Density of basic } \\
\text { sites }\left(\mathrm{g}^{-1} \text { catalyst }\right)\end{array}$ & $k\left(\mathrm{~min}^{-1}\right)$ & $t_{1 / 2}(\mathrm{~min})$ & $R^{2}$ \\
\hline $\mathrm{ZnO}-1$ & 16.35 & $8.95 \times 10^{13}$ & $2.71 \times 10^{-2}$ & 27.5 & 0.999 \\
$\mathrm{ZnO}-2$ & 8.72 & $1.03 \times 10^{14}$ & $4.71 \times 10^{-2}$ & 17.5 & 0.998 \\
$\mathrm{ZnO}-3$ & 8.35 & $7.99 \times 10^{13}$ & $3.87 \times 10^{-2}$ & 22.5 & 0.997 \\
$\mathrm{ZnO}-4$ & 12.36 & $7.87 \times 10^{13}$ & $3.24 \times 10^{-2}$ & 24.0 & 0.995 \\
$\mathrm{ZnO}-5$ & 6.90 & $1.06 \times 10^{14}$ & $2.03 \times 10^{-2}$ & 40 & 0.986 \\
\hline
\end{tabular}

On the other hand, the electrons in the conduction band can reduce $\mathrm{O}_{2}$ to produce superoxide radicals $\mathrm{O}_{2}{ }^{*}$ (Equation 9), which subsequently react with hydrogen ions generated through water splitting to form hydroperoxyl $\mathrm{HO}_{2}{ }^{\circ}$ (Equation 10) radical and other reactive oxygen species such as $\mathrm{H}_{2} \mathrm{O}_{2}$ and $\mathrm{OH}^{*}$ (Equation 11 and 12):

$$
\begin{aligned}
& \mathrm{O}_{2}+e_{C B}^{-} \rightarrow \mathrm{O}_{2}^{\bullet} \\
& \mathrm{O}_{2}^{\bullet}+\mathrm{H}^{+} \rightarrow \mathrm{HO}_{2}^{\bullet}
\end{aligned}
$$

$2 \mathrm{OH}^{\bullet} \rightarrow \mathrm{H}_{2} \mathrm{O}_{2}$

$$
\mathrm{H}_{2} \mathrm{O}_{2}+e_{\mathrm{CB}}^{-} \rightarrow \mathrm{HO}^{\bullet}+\mathrm{OH}^{-}
$$

Photocatalytic degradation of most of the organic compounds in a heterogeneous system follows first-order kinetics (Serrano et al., 2004; Yadav et al., 2007), for which the steady-state photocatalytic rate can be expressed through Langmuir-Hinshelwood relation. The photocatalytic degradation of $\mathrm{MB}$ by the $\mathrm{ZnO}$ nanostructures grown at different reaction conditions 
obeyed the pseudo-first-order kinetics. The photo degradation rate of MB for low initial concentration can be expressed as:

$$
\ln C=\ln C_{0}-k t
$$

Or:

$$
\ln \left(C / C_{0}\right)=-k t
$$

where, $k$ is the pseudo-first-order rate constant, $C_{0}$ is the initial concentration of $\mathrm{MB}$ in reaction solution and $C$ is the $\mathrm{MB}$ concentration in reaction solution after time $t$ of UV illumination.

The reduction of $\mathrm{MB}$ content in catalytic solution upon UV irradiation for a typical sample $(\mathrm{ZnO}-2)$, the plots of $C / C_{0}$ versus irradiation time and $\ln \left(C / C_{0}\right)$ versus irradiation time for all the nanostructures are shown in Fig. 6. The linear relationship between $\ln \left(C / C_{0}\right)$ and the UV illumination time (Fig. 6C) clearly demonstrates the photo-degradation process follows first-order kinetics. The values of the pseudo-first-order rate constant $k$ estimated from the linear regression curves (linear fits), the estimated values of $t_{1 / 2}$ (dye half-life) and corresponding correlation coefficients $\left(R^{2}\right)$ for the $\mathrm{ZnO}$ nanostructures are presented in Table 2 . We can see that the sample $\mathrm{ZnO}-2$ prepared using $\mathrm{CTAB}$ at $\mathrm{ZnAc:NaOH}$ $=1: 3$ molar ratio has highest rate constant $\left(k=4.71 \times 10^{-2}\right.$ $\left.\min ^{-1}\right)$ and lowest $t_{1 / 2}(17.5 \mathrm{~min})$ among the nanostructures, indicating their excellent photocatalytic activity for MB degradation. From the data presented in Table 1 and 2, we can see that all the nanostructures have near-stoichiometric composition with minor variation of average crystallite size (32-38 $\mathrm{nm})$. However, due to morphological diversity, the BET surface area and BET pore size in the nanostructures varied. As apart from surface area, pore size and crystallite size, the photocatalytic activity of metal-oxide nanostructures depend on the density of basic $\left(\mathrm{OH}^{-}\right)$or acid sites at their surfaces, a quick and fast quantitative measurement of basic sites was performed following the method described by Corro et al. (2014). As the nanostructures were fabricated in highly basic conditions, the density of basic sites at their surface was very high (Table 2), which is responsible for their enhanced dye adsorption capacity.
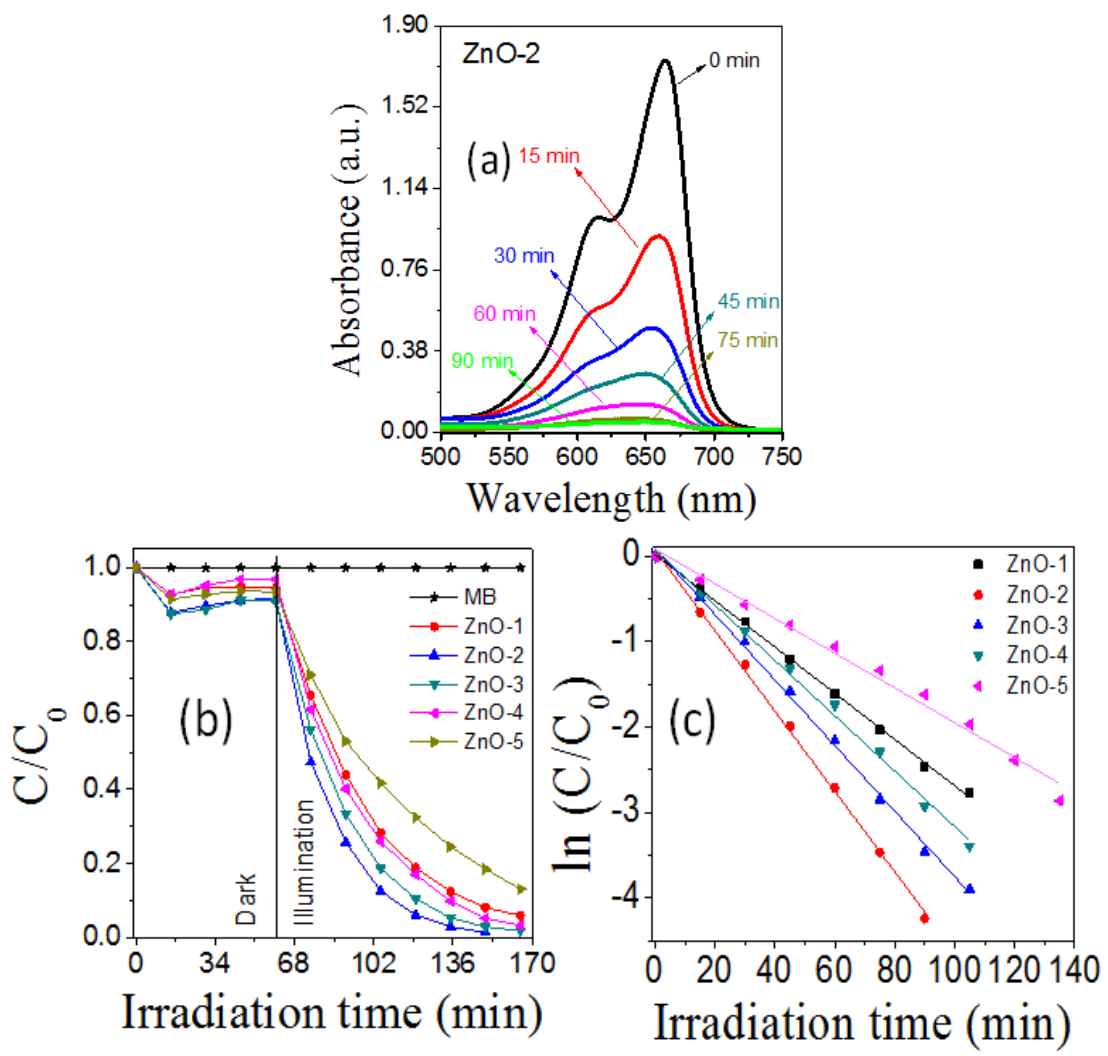

Fig. 6. (a) MB absorption spectra used to monitor the photocatalytic degradation rate for the sample $\mathrm{ZnO}$-2, (b) photo-degradation rate of $\mathrm{MB}$ over the $\mathrm{ZnO}$ nanostructures prepared at different conditions and (c) $\ln (\mathrm{C} / \mathrm{C} 0) \mathrm{Vs}$ irradiation time plots used to evaluate the degradation rate-constants. The MB degradation behavior under UV illumination has also been included in (b) to show that there is no photo-degradation of MB in absence of catalyst 
While all the nanostructures fabricated in our soft mechanochemical process present high efficiency for MB degradation under UV illumination, the sample $\mathrm{ZnO}-2$ prepared at $\mathrm{ZnAc}: \mathrm{NaOH}=1: 3$ in presence of $\mathrm{CTAB}$ revealed best MB degradation performance. The nanostructures of sample $\mathrm{ZnO}-2$ contain highest surface basic sites, bigger average crystallite size, smaller average pore diameter and moderate BET surface area (Table 1 and 2). Therefore, it is very difficult to associate their higher photocatalytic performance to any one of these texture parameters specifically, as have been practiced in a vast number of published works. Considering the morphology and growth habit of all the nanostructures fabricated in this study, it seems, the high photocatalytic performance of the well dispersed rod-like nanostructures of $\mathrm{ZnO}-2$ sample is due to the high exposure of their non-polar facets, which do not adsorb MB molecules as strongly as polar surfaces.

A strong adsorption of cationic dye over polar surface of $\mathrm{ZnO}$ probably blocks the penetration of exciting UV radiation, responsible for generating electron-hole pairs at semiconductor surface. On the other hand, the electron mobility in flater $\mathrm{ZnO}$ surface such as nanopetals is expected to be higher than other surfaces due to their directional and uninterrupted conduction channel. The high electron mobility of semiconductor surface would increase the recombination rate of photo-generated electrons and holes, which is unfavorable for photcatalytic process.

\section{Conclusion}

In summary, $\mathrm{ZnO}$ nanostructures of different morphologies could be fabricated in large scale and controlled manner through solvent-free mechanochemical process by controlling the precursor to hydrolyzer molar ratio and suitable choice of surfactant. Apart from its cheapness, the process can produce nearstoichiometric $\mathrm{ZnO}$ nanostructures with enhanced photocatalytic performance for cationic dye degradation under UV illumination. The high concentration of basic sites at the surface of the nanostructures is the principal responsible for their high photocatalytic activity. However, due to polar nature of $\mathrm{ZnO}$ crystals, the orientation of crystal facets exposed to the reaction mixture also plays a significant role on their photocatalytic performance. The $\mathrm{ZnO}$ nanostructures fabricated by our soft mechanochemical synthesis process can mineralize $50 \%$ of MB molecules in aqueous solution as fast as in $17.5 \mathrm{~min}$. The large scale production ability, low cost processing and high dye degradation ability of the fabricated nanostructures suggest the solvent-free mechanochemical process is extremely attractive for fabricating $\mathrm{ZnO}$ nanostructures for dye removal from industrial effluents.

\section{Acknowledgement}

The work was financially supported by VIEP-BUAP and CUVyTT-BUAP and CONACYT, Mexico, through the grants \# VIEP/EXC/2015-205, DITCo-2015-38 and INFR-2011-1-163153, respectively.

\section{Author's Contributions}

Raúl Sánchez Zeferino: Synthesized the $\mathrm{ZnO}$ nanostructures.

Jesús Alberto Ramos Ramón: Performed surface area and texture characterization of the nanostructures.

Eunice De Anda Reyes: Performed the photocatalytic characterization of the nanostructures.

Rutilo Silva González: Performed morphology and composition evaluation of the nanostructures.

Umapada Pal: Planned and coordinated the research, analyzed the results and wrote the article.

\section{Ethics}

This article is original and contains unpublished material. The corresponding author confirms that all of the other authors have read and approved the manuscript and no ethical issues involved.

\section{References}

An, S., S. Partk, H. Ko, C. Jin and W.I. Lee et al., 2013. Enhanced gas sensing properties of branched $\mathrm{ZnO}$ nanowires. Thin Solid Films, 547: 241-245.

DOI: $10.1016 /$ j.tsf.2013.02.021

Anand, K., S. Varghese and T. Kurian, 2014. Synthesis of $\mathrm{ZnO}$ nanorods through mechanochemical route: A solvent free approach. Int. J. Theo. Applied Sci., 6: 87-93.

Bai, S., L. Chen, D. Li, W. Yang and P. Yang et al., 2010. Different morphologies of $\mathrm{ZnO}$ nanorods and their sensing property. Sens. Actuators B, 146: 129-137. DOI: 10.1016/j.snb.2010.02.011

Barrett, E.P., L.G. Joyner and P.P. Halenda, 1951. The determination of pore volume and area distributions in porous substances. I. Computations from nitrogen isotherms. J. Am. Chem. Soc., 73: 373-380. DOI: $10.1021 /$ ja01145a126

Brunauer, S., P.H. Emmett and E. Teller, 1938. Adsorption of gases in multimolecular layers. J. Am. Chem. Soc., 60: 309-319. DOI: $10.1021 /$ ja01269a023

Chen, D., X. Jiao and G. Cheng, 2000. Hydrothermal synthesis of zinc oxide powders with different morphologies. Solid State Commun., 113: 363-366. DOI: 10.1016/S0038-1098(99)00472-X 
Chen, X., F. Zhang, Q. Wang, X. Han and X. Li et al., 2015. The synthesis of $\mathrm{ZnO} / \mathrm{SnO}_{2}$ porous nanofibers for dye adsorption and degradation. Dalton Trans., 44: 3034-3042. DOI: 10.1039/C4DT03382E

Corro, G., F. Bañuelos, E. Vidal and S. Cebada, 2014. Measurements of surface acidity of solid catalysts for free fatty acids esterification in jatropha curcas crude oil for biodiesel production. Fuel, 115: 625-628. DOI: 10.1016/j.fuel.2013.07.060

Cullity, B.D. and S.R. Stock, 1956. Elements of X-Ray Diffraction. 1st Edn., Addison-Wesley Publishing Company, Inc., Massachusetts, ISBN-10: 0201610914, pp: 514.

Cuscó, R., E. Alarcón-Lladó, J. Ibáñez, L. Artus and J. Jimenez et al., 2007. Temperature dependence of Raman scattering in ZnO. Phys. Rev. B, 75: 165202-11. DOI: 10.1103/PhysRevB.75.165202

Hahn, Y.B., R. Ahmad and N. Tripathy, 2012. Chemical and biological sensors based on metal oxide nanostructures. Chem. Commun., 48: 10369-10385. DOI: $10.1039 / \mathrm{C} 2 \mathrm{CC} 34706 \mathrm{G}$

He, J.H., J.H. Hsu, C.W. Wang, H.N. Lin and L.J. Chen et al., 2006. Pattern and feature designed growth of $\mathrm{ZnO}$ nanowire arrays for vertical devices. J. Phys. Chem. B, 110: 50-53. DOI: 10.1021/jp055180j

Hilger, I. and W.A. Kaiser, 2012. Iron oxide-based nanostructures for MRI and magnetic hyperthermia. Nanomedicine, 7: 1443-1459.

DOI: $10.2217 / \mathrm{NNM} .12 .112$

Huang, J.R., Y.J. Wu, C.P. Gu, M.H. Zhai and K. Yu et al., 2010. Large-scale synthesis of flowerlike $\mathrm{ZnO}$ nanostructure by a simple chemical solution route and its gas-sensing property. Sens. Actuators B, 146: 206-212. DOI: 10.1016/j.snb.2010.02.052

Hsu, Y.F., Y.Y. Xi, K.H. Tam, A.B. Djurišić and J. Luo et al., 2008. Undoped p-type $\mathrm{ZnO}$ nanorods synthesized by a hydrothermal method. Adv. Funct. Mater., 18: 1020-1030. DOI: $10.1002 / \mathrm{adfm} .200701083$

Jalali, N., J. Briscoe, P. Woolliams, M. Stewart and P.M. Weaver et al., 2013. Passivation of zinc oxide nanowires for improved piezoelectric energy harvesting devices. J. Phys., 476: 012131-1-012131-6. DOI: $10.1088 / 1742-6596 / 476 / 1 / 012131$

Jang, J.M., S.D. Kim, H.M. Choi, J.Y. Kim and W.G. Jung, 2009. Morphology change of self-assembled $\mathrm{ZnO} 3 \mathrm{D}$ nanostructures with different $\mathrm{pH}$ in the simple hydrothermal process, Mater. Chem. Phys., 113: 389-394.

DOI: 10.1016/j.matchemphys.2008.07.108

Jia, X., M. Tian, Y. Liu, X. Wu and H. Song, 2015. In situ precipitation preparation of $\mathrm{ZnO}$ hollow spheres and their photocatalysis and gas sensing properties. Appl. Phys. A, 119: 1179-1185.

DOI: $10.1007 / \mathrm{s} 00339-015-9088-1$
Khosla, E., S. Kaur and P.N. Dave, 2015. Ionic dye adsorption by zinc oxide nanoparticles. Chem. Ecol., 31: 173-185. DOI: 10.1080/02757540.2014.907281

Kumar, R., O. Al-Dossary, G. Kumar and A. Umar, 2015. Zinc oxide nanostructures for $\mathrm{NO}_{2}$ GasSensor applications: A review. Nano-Micro Lett., 7: 97-120. DOI: $10.1007 / \mathrm{s} 40820-014-0023-3$

Lorenz, H., M. Friedrich, M. Armbrüster, B. Klötzer and S. Penner, 2013. $\mathrm{ZnO}$ is a $\mathrm{CO}_{2}$ selective steam reforming catalyst. J. Catal., 297: 151-154. DOI: $10.1016 /$ j.jcat.2012.10.003

Li, F., L. Hu, Z. Li and X. Huang, 2008. Influence of temperature on the morphology and luminescence of $\mathrm{ZnO}$ micro and nanostructures prepared by CTABassisted hydrothermal method. J. Alloy Comp., 465: L14-L19. DOI: 10.1016/j.jallcom.2007.11.009

Lu, A.H., D. Zhao and Y. Wan, 2012. Nanocasting: A Versatile Strategy for Creting Nanostructured Porous Materials. 1st Edn., RSC Publishing, ISBN-13: 978-0-85404-188-6.

Matsamura, Y. and H. Ishibe, 2009. Suppression of $\mathrm{CO}$ by-production in steam reforming of methanol by addition of zinc oxide to silica-supported catalyst. J. Catal., 268: 282-289. DOI: $10.1016 /$ j.jcat.2009.09.026

Morales Flores, N., U. Pal, R. Galeazzi and A. Sandoval, 2014. Effects of morphology, surface area and defect contento $\mathrm{n}$ the photocatalytic dye degradation performance of $\mathrm{ZnO}$ nanostructures. RSC Adv., 4: 41099-41110. DOI: 10.1039/c4ra04552j

Morkoç, H. and Ü. Özgür, 2008. Zinc Oxide: Fundamentals, Materials and Device Technology. 1st Ed., John Wiley and Sons, Germany, ISBN-10: 3527623957, pp: 500.

Ni, Y.H., X.W. Wei, X. Ma and J.M Hong, 2005. CTAB assited one-pot hydrothermal synthesis of columnar hexagonal-shaped $\mathrm{ZnO}$ cystals. J. Cryst. Growth, 283: 48-56. DOI: 10.1016/j.jcrysgro.2005.05.048

Norman, V.J., 1970. The adsorption of dyes on zinc oxide: The effect of non-stoicheiometry. Aust. J. Chem., 23: 2171-2176. DOI: 10.1071/CH9702171

Olson, D.C., Y.J. Lee, M.S. White, N. Kopidakis and S.E. Shaheen et al., 2007. Effect of polymer processing on the performance of poly(3-hexylthiophene)/ZnO nanorod photovoltaic devices. J. Phys. Chem. C, 111: 16640-16645. DOI: 10.1021/jp0757816

Pan, T.C., Y.C. Chen, C.C. Hsieh, C.H. Lin and C.Y. Su et al., 2014. Ultrasonic sensing device with $\mathrm{ZnO}$ piezoelectric nanorods by selectively electrospraying method. Sensors Actuators A, 216: 318-327. DOI: 10.1016/j.sna.2014.05.024

Ruiz Peralta, M.L., U. Pal and R. Sánchez Zeferino, 2012. Photoluminescence (PL) quenching and enhanced photocatalytic activity of Au-decorated $\mathrm{ZnO}$ nanorods fabricated through microwaveassisted chemical synthesis. ACS App. Mater. Interfaces, 4: 4807-4816. DOI: 10.1021/am301155u 
Serrano, J., A.H. Romero, F.J. Manjon, R. Lauck and M. Cardona et al., 2004. Pressure dependence of the lattice dynamics of $\mathrm{ZnO}$ : An ab initio approach. Phys. Rev. B: Condens. Matter Mater. Phys., 69: 094306. DOI: 10.1103/PhysRevB.69.094306

Shao, S., P. Jia, S. Liu and W. Bai, 2008. Stable field emission from rose-like zinc oxide nanostructures synthesized through a hydrothermal route. Mater. Lett., 62: 1200-1203. DOI: 10.1016/j.matlet.2007.08.049

Shen, L., L. Guo, N. Bao and K. Yanagisawa, 2003. Salt-assited solid-state chemical reaction. Synthesis of $\mathrm{ZnO}$ nanocrystals. Chem. Lett., 32: 826-827. DOI: $10.1246 / \mathrm{cl} .2003 .826$

Solanki, P.R., A. Kaushik, V.V. Agrawal and B.D. Malhotra, 2011. Nanostructured metal oxidebased biosensors. NPG Asia Mater., 3: 17-24. DOI: 10.1038 /asiamat.2010.137

Spencer, M.S., 1999. The role of zinc oxide in $\mathrm{Cu} / \mathrm{ZnO}$ catalysts for methanol synthesis and water-gas shift reaction. Top. Catal., 8: 259-266. DOI: 10.1023/A:1019181715731

Tak, Y. and K. Yong, 2005. Controlled growth of wellaligned $\mathrm{ZnO}$ nanorod array using a novel solution method. J. Phys. Chem. B, 109: 19263-19269. DOI: $10.1021 /$ jp0538767

Tsuzuki, T. and P.G. McCormick, 2001. ZnO nanoparticles synthesized by mechanochemical processing. Scripta Mater., 44: 1731-1734. DOI: 10.1016/S1359-6462(01)00793-X

Tsuzuki, T. and P.G. McCormick, 2004. Mechanochemical synthesis of nanoparticles. J. Mater. Sci., 39: 5143-5146. DOI: 10.1023/B:JMSC.0000039199.56155.f9

Valerini, D., A.P. Caricato, M. Lomascolo, F. Romano and A. Taurino et al., 2008. Zinc oxide nanostructures grown by pulsed laser deposition. Appl. Phys. A, 93: 729-733. DOI: 10.1007/s00339-008-4703-z

Wahab, R., Y.S. Kim and H.S. Shin, 2009. Synthesis, characterization and effect of $\mathrm{pH}$ variation on zinc oxide nanostructures. Mater. Trans., 50: 2092-2097. DOI: 10.2320/matertrans.M2009099

Wang, J.X., X.W. Sun, Y. Yang, H. Huang and Y.C. Lee et al., 2006. Hydrothermally grown oriented $\mathrm{ZnO}$ nanorod arrays for gas sensing applications. Nanotechnology, 17: 4995-4998. DOI: $10.1088 / 0957-4484 / 17 / 19 / 037$

Wang, L., Y. Kang, X. Liu, S. Zhang and W. Huang et al., 2012. ZnO nanorod gas sensor for ethanol detection. Sensors Actuators B, 162: 237-243. DOI: $10.1016 /$ j.snb.2011.12.073

Wang, L., X. Zhang, S. Zhao, G. Zhou and Y. Zhou et al., 2005. Synthesis of well-aligned $\mathrm{ZnO}$ nanowires by simple physical deposition on c-oriented $\mathrm{ZnO}$ thin films without catalysts or additives. Appl. Phys. Lett., 86: 024108-1-024108-3. DOI: 10.1063/1.1851607
Wang, X., C.J. Summers and Z.L. Wang, 2004. Largescale hexagonal-patterned growth of aligned zno nanorods for nano-optoelectronics and nanosensor arrays. Nano Lett., 4: 423-426.

DOI: $10.1021 / \mathrm{nl} 035102 \mathrm{c}$

Wang, X., Y. Ding, C.J. Summers and Z.L. Wang, 2004. Large-scale synthesis of six-nanometer-wide $\mathrm{ZnO}$ nanobelts. J. Phys. Chem. B, 108: 8773-8777. DOI: $10.1021 /$ jp048482e

Wang, Z.L. and J. Song, 2006. Piezoelectric nanogenerators based on zinc oxide nanowire arrays. Science, 312: 242-246. DOI: 10.1126/science. 1124005

Xie, J., P. Li, Y.J. Wang and Y. Wei, 2009. Synthesis of needle- and flower-like $\mathrm{ZnO}$ microstructures by a simple aqueous solution route. J. Phys. Chem. Solids, 70: 112-116. DOI: $10.1016 /$ j.jpcs.2008.09.014

Xie, J., Y. Li, W. Zhao, L. Bian and Y. Wei, 2011. Simple fabrication and photocatalytic activity of $\mathrm{ZnO}$ particles with different morphologies. Powder Technol., 207: 140-144. DOI: $10.1016 /$ j.powtec.2010.10.019

Xu, J., Q. Pan, Y. Shun and Z. Tian, 2000. Grain size control and gas sensing properties of $\mathrm{ZnO}$ gas sensor. Sensors Actuators B, 66: 277-279. DOI: $10.1016 / \mathrm{S} 0925-4005(00) 00381-6$

Yadav, H.K., K. Sreenivas, R.S. Katiyar and V. Gupta, 2007. Defect induced activation of Raman silent modes in rf co-sputtered $\mathrm{Mn}$ doped $\mathrm{ZnO}$ thin films. J. Phys. D, 40: 6005-6009. DOI: $10.1088 / 0022-3727 / 40 / 19 / 034$

Yan, L., Y. Li, Z.X. Deng, J. Zhuang and X. Sun, 2001. Surfactant-assisted hydrothermal synthesis of hydroxyapatite nanorods. Int. J. Inorg. Mater., 3: 633-637. DOI: 10.1016/S1466-6049(01)00164-7

Yang, L., X. Kong, J. Wang, M. Pan and W. Yang et al., 2015. Synthesis and photocatalytic performance of $\mathrm{ZnO}$ hollow spheres and porous nanosheets. J. Mater. Sci. DOI: 10.1007/s10854-015-3738-0

Zhang, Z., M. Lu, H. Xu and W.S. Chin, 2007. Shapecontrolled synthesis of zinc oxide: A simple method for the preparation of metal oxide nanocrystals in non-aqueous medium. Chem. Euro. J., 13: 632-638. DOI: 10.1002/chem.200600293

Zimmler, M.A., D. Stichtenoth, C. Ronning, W. Yi and V. Narayanamurti et al., 2008. Scalable fabrication of nanowire photonic and electronic circuits using spin-on glass. Nano Lett., 8: 1695-1699. DOI: $10.1021 / \mathrm{n} 1080627 \mathrm{w}$ 